\title{
The role of interleukin-24 in atopic dermatitis
}

\author{
Yen Hai Vu间, Masutaka Furue ${ }^{1,2^{*}}\left(\mathbb{0}\right.$, Alessandro Minardo $^{1,2}(\mathbb{0})$ \\ ${ }^{1}$ Department of Dermatology, Graduate School of Medical Sciences, Kyushu University, Fukuoka 812-8582, Japan \\ ${ }^{2}$ Research and Clinical Center for Yusho and Dioxin, Kyushu University, Fukuoka 812-8582, Japan
}

*Correspondence: Masutaka Furue, Department of Dermatology, Graduate School of Medical Sciences, Kyushu University, Fukuoka 812-8582, Japan. furue@dermatol.med.kyushu-u.ac.jp; furuemasutaka00@yahoo.co.jp

Academic Editor: Dominique J Charron, Hôpital Saint-Louis, France; Reem Al-Daccak, Hôpital Saint-Louis, France

Received: January 5, 2021 Accepted: January 26, 2021 Published: April 30, 2021

Cite this article: Vu YH, Furue M, Tsuji G. The role of interleukin-24 in atopic dermatitis. Explor Immunol. 2021;1:4-15. https:// doi.org/10.37349/ei.2021.00002

\begin{abstract}
Atopic dermatitis (AD) is characterized by skin barrier disruption, type 2 immune dysregulation, chronic pruritus, and abnormal colonization by Staphylococcus aureus (S. aureus). Tapinarof, an aryl hydrocarbon receptor modulator, has been demonstrated to attenuate the development of AD in clinical studies. Recently, we found that tapinarof upregulated the expression of filaggrin and loricrin, which are essential proteins in skin barrier functions. Paradoxically, tapinarof induced interleukin (IL)-24 secretion by normal human keratinocytes. IL-24 is produced by T helper 2 lymphocytes and keratinocytes following stimulation by type 2 cytokines, and IL-24 is upregulated in the skin of patients with AD. Furthermore, IL-24 contributes to skin barrier disruption and hyperplasia in $\mathrm{AD}$, and it may exacerbate skin inflammatory responses, itch, and $S$. aureus infection. In this review, we summarized the current findings regarding the detrimental role of IL-24 in $\mathrm{AD}$, thereby suggesting that co-treatment of tapinarof with therapeutics that block IL-24 signaling may represent a promising strategy for managing AD.
\end{abstract}

\section{Keywords}

Atopic dermatitis, interleukin-24, aryl hydrocarbon receptor, tapinarof, Janus kinase inhibitor, dupilumab

\section{Introduction}

Atopic dermatitis (AD), a common inflammatory skin disease, is associated with significant physiopsychological and socioeconomic burdens in affected patients [1]. AD is characterized by skin barrier disruption, type 2 immune dysregulation, chronic pruritus, and abnormal colonization by Staphylococcus aureus (S. aureus) [2,3]. Among the emerging therapeutic agents for AD, topical tapinarof, an aryl hydrocarbon receptor (AHR) modulator, has been revealed to attenuate disease activity clinically [4, 5, 6]. Recently, we demonstrated that tapinarof upregulated the expression of filaggrin (FLG) and loricrin (LOR), which are important skin barrier-related proteins. Paradoxically, tapinarof stimulated human keratinocytes to secrete interleukin (IL)-24 [7]. Although IL-24 expression is upregulated in the epidermis in patients with $\mathrm{AD}[8,9]$, its implications in the pathogenesis of AD remain poorly investigated. To date, IL-24 has 
been extensively studied in cancer and demonstrated to induce apoptosis in several different cancer cells without harming normal cells [10]. In addition, IL-24 was implicated to play a pivotal pathogenic role in inflammatory diseases such as psoriasis, arthritis, and inflammatory bowel disease. Furthermore, IL-24 was shown to have a potential role in type 2 inflammation-related allergic diseases, such as allergic rhinitis, asthma, and $\mathrm{AD}$ [11]. IL-24 contributes to skin barrier disruption and hyperplasia in AD and may promote skin inflammatory responses, itch, and S. aureus infection. Therefore, IL-24 may exacerbate AD lesions. In this review, we summarized the importance of IL-24 in the pathogenesis and treatment of AD.

\section{IL-24 expression in AD}

IL-24 gene overexpression was detected in the lesional skin of patients with AD [12]. IL-24 protein expression was upregulated from the basal layer to the spinous cell layer, but not in the granular and cornified layers, in the epidermis of skin tissues from patients with AD [9]. In murine AD models, IL-24 expression was enhanced in the epidermis of mite-treated mice [9] and $I L-4$ transgenic mice [8]. No data have been presented regarding IL-24 serum levels in patients with AD; however, the serum levels of this cytokine did not differ between $I L-4$ transgenic and wild-type mice, suggesting the local role of IL-24 in AD pathophysiology [8].

\section{Sources of IL-24 in the skin}

IL-24 can be produced by immune cells, including monocytes, macrophages, mast cells, natural killer cells, and $\mathrm{T}$ and $\mathrm{B}$ lymphocytes, as well as non-immune cells, such as keratinocytes and melanocytes, in response to certain stimuli $[10,11]$ (Figure 1 ).

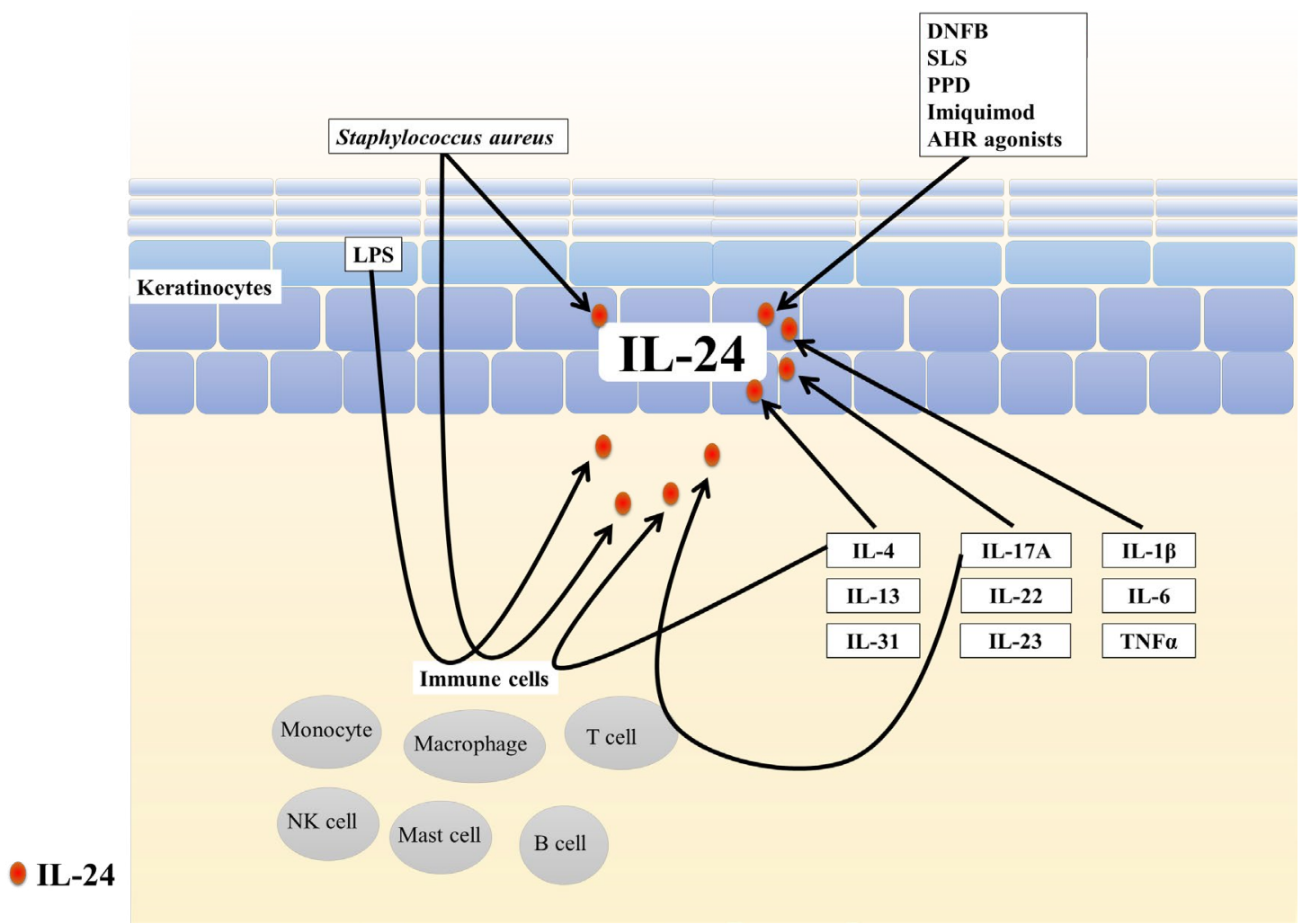

Figure 1. Sources of IL-24 in the skin. IL-24 can be produced by immune cells, including monocytes, macrophages, mast cells, natural killer cells, and T and B lymphocytes, and non-immune cells such as keratinocytes in response to certain stimuli. DNFB: 2,4-dinitrofluorobenzene; SLS: sodium lauryl sulfate; PPD: paraphenylenediamine; LPS: lipopolysaccharide

\section{Chemical stimuli}

2,4-Dinitrofluorobenzene, which was revealed to induce AD-like pathology in mice [13], upregulates IL-24 expression in murine skin [14]. Sodium lauryl sulfate (a chemical irritant in human skin) stimulates IL-24 production in normal human keratinocytes [15]. IL-24 was overexpressed in an allergic contact dermatitis model induced by paraphenylenediamine exposure, in human and mouse skin [16]. IL-24 expression was 
also increased in imiquimod-stimulated HaCaT cells, an immortalized keratinocyte cell line [17]. Phorbol myristate acetate and ionomycin also induced $I L-24$ in T helper 2 (Th2) lymphocytes [10].

Some studies determined that IL-24 is the target cytokine of some oxidative environmental AHR agonists, such as 2, 3, 7, 8-tetrachloro-dibenzo- $p$-dioxin (TCDD), benzo(a)pyrene, particulate matter, and ultraviolet B irradiation, in primary human chorionic stromal cells, human lung adenocarcinoma cells, normal human bronchial epithelial cells, and normal keratinocytes, respectively [15, 18-21]. In line with these findings, we recently demonstrated that tapinarof, an antioxidative AHR modulator [22], stimulated normal human epidermal keratinocytes to generate IL-24 protein, whereas AHR depletion significantly attenuated the upregulation of IL-24 induced by tapinarof [7]. The AHR-binding sequence (GCGTG) is present in the promoter region of the $I L-24$ gene $[18,23]$. These results indicated that AHR activation plays a vital role in the mechanism of AHR ligand-mediated IL-24 induction.

\section{Microbial stimuli}

Staphylococcal strains, both pathogenic (S. aureus) and commensal strains (S. epidermidis and S. saprophyticus), induce IL-24 mRNA and protein expression in human peripheral blood mononuclear cells [24]. The expression of $I L-24$ was also upregulated by S. aureus infection in human keratinocytes [25].

Lipopolysaccharide, a microbial product and Toll-like receptor activator, stimulates monocytes [26], macrophages [24, 27], and activated T cells [28] to produce IL-24. Pellino 1 ubiquitin E3 ligase is activated by innate pattern-recognition receptors such as Toll-like receptors. Overexpression of Pellino 1 induced a significant increase in the expression of $I L-24$ in HaCaT cells [29].

\section{The Th2-type cytokines IL-4, IL-13, and IL-31}

It is widely accepted that the Th2 cytokines IL- 4 and IL-13 are driving factors in AD pathogenesis, as the blockade of IL-4/IL-13 signaling by dupilumab is effective in patients with severe AD [30]. The $I L-24$ gene has been identified as a dominant Th2 lineage-specific gene [31].

IL-4 increases IL-24 expression in normal human keratinocytes [9, 15], human monocytes [27], and T lymphocytes [28]. $I L-24$ expression is increased in the inflammatory skin lesions of $I L$-4-transgenic mice compared with that in wild-type mice [8]. IL-4 functions synergistically with IL-2 or lipopolysaccharide to induce $I L-24$ expression in natural killer (NK) cells and macrophages [32].

IL-13 upregulates periostin production via signal transducer and activator of transcription 6 (STAT6) activation, and periostin enhances IL-24 production in human keratinocytes [9]. STAT6 is critical for IL-4/ IL-13-induced IL-24 expression [9, 31]. In Th2 cells, the transcription factors STAT6, GATA3, and the activator protein-1 (AP-1) family member c-Jun were revealed to participate in the regulation of IL-24 production [31, 33, 34].

IL-31 stimulates keratinocytes to produce $I L$-24. The inhibition of Janus kinase (JAK), p38, and extracellular signal-regulated kinases (ERKs), but not c-Jun N-terminal kinases or phosphoinositide 3-kinases, interfere with the upregulation of IL-24 induced by IL-31 [35].

\section{Cytokines IL-17A, IL-22, and IL-23}

IL-17A upregulated $I L-24$ expression in normal human keratinocytes [15, 36]. Chong et al. [37] found that IL-17A induced IL-24 production in Th17 cells. Considering that IL-17A signals through the nuclear factor kappa-light-chain-enhancer of activated B cells (NF- $\kappa$ B) pathway [38] and two potential NF- $\kappa$ B binding sites are present in the IL-24 gene, the researchers proposed that the binding of IL-17A to its receptor activates NF- $\kappa B$ signaling, leading to the transcription of $I L-24$ in Th17 cells [37]. IL-22 induced the production of IL-24 in normal human keratinocytes [15, 39, 40]. IL-17 synergistically induced the expression of $I L-24$ in keratinocytes with IL-22 or tumor necrosis factor alpha (TNF $\alpha)[36,39,40]$. The injection of IL-23 into mouse skin elevated $I L-24$ expression [41]. 


\section{Pro-inflammatory cytokines IL-1 $\beta$, IL-6, and TNF $\alpha$}

IL-1 $\beta$ stimulation induced IL-24 protein secretion in human keratinocytes $[14,15,42]$. Inhibition of the p38 mitogen-activated protein kinase (MAPK) pathway was reported to significantly decrease IL-1 $\beta$-induced $I L-24$ expression by reducing IL-24 mRNA stabilization, suggesting that p38 MAPK regulates $I L-24$ gene expression at the post-transcriptional level [43-45].

IL-6 induced the production of IL-24 in human keratinocytes $[15,46]$. Suppressor of cytokine signaling-3 (SOCS3) inhibited JAK1, JAK2, and tyrosine protein kinase 2 but not JAK3, and it downregulated STAT3 activation [47]. The dysregulation of SOCS3 resulted in excess STAT3 activation in response to IL-6, leading to the upregulation of $I L-24$ expression by keratinocytes [48]. In an experimental model, physical stimulation (i.e. shaving) induced $I L-24$ expression at the site of stimulation in SOCS3 deficient mice, but not in control mice [48].

TNF $\alpha$ also upregulated IL-24 expression in keratinocytes [15, 49, 50-52]. Anti-TNF $\alpha$ antibody treatment almost completely inhibited IL-24 expression in psoriatic skin, indicating that IL-24 production is mainly driven by TNF $\alpha$ in psoriasis. Reactive oxygen species-activated ERK signaling was illustrated to mediate the upregulation of IL-24 in response to TNF $\alpha$ stimulation in keratinocytes [52].

\section{IL-24 signal transduction}

The human $I L-24$ gene is located on chromosome 1q32-33. IL-24, together with IL-19, IL-20, IL-22, and IL-26, belongs to the IL-20 cytokine subfamily and IL-10 family, which further includes IL-10, IL-28, and IL-29. IL-24 signals through two heterodimeric receptors: IL-20R1/IL-20R2 and IL-22R1/IL-20R2. Notably, IL-24 shares the same two receptors with IL-20 and shares IL-20R1/IL-20R2 with IL-19 receptor, indicating that IL-24 may have partially overlapping biological activities with IL-19 and IL-20 [53].

In most immune cells, only IL-20R2 is expressed, whereas IL-20R1 and IL-22R1 are undetectable [14]. By contrast, these three receptor subunits are all expressed in several non-hematopoietic cells, such as keratinocytes and bronchial epithelial cells [14, 54-56]. Interestingly, IL-24-induced STAT3 activation was detected in keratinocytes but not in peripheral blood mononuclear cells, probably because of the lack of IL-24 receptor expression in immune cells [14]. These findings suggest a preferential effect of IL-24 on nonimmune cells, including keratinocytes.

IL-24 binds to its receptor, leading to specific activation of JAK1/tyrosine protein kinase 2-STAT3 and ERK1/2 MAPK signaling pathways in keratinocytes $[14,15,53,54,56]$. In addition, STAT3 activation in keratinocytes is elevated in the skin of patients with $\mathrm{AD}[9]$.

\section{Role of IL-24 in AD}

As mentioned previously, the cardinal features of AD include skin barrier disruption, immune dysregulation, chronic pruritus, and abnormal skin colonization by pathogens. The following sections will discuss the role of IL-24 in AD concerning these features (Figure 2).

\section{IL-24 and keratinocyte differentiation/proliferation}

Skin barrier dysfunction is a critical factor in the pathogenesis of AD. The disrupted barrier permits allergen penetration, accelerating immune response, pruritus, and trans-epidermal water loss [3]. In the epidermal barrier, FLG and LOR, markers of late differentiation of keratinocytes [3], are essential barrier-related proteins. They aggregate and align keratin bundles, contributing to skin barrier strength and integrity. In addition, FLG degradation products form a natural moisturizing factor, which has a role in skin hydration and barrier function [57]. In AD, overexpressed IL-4/IL-13 repressed FLG and LOR expression, leading to the impaired terminal differentiation and barrier function of the epidermis [2, 7]. IL-4/IL-13-mediated barrier dysfunction may be, at least in part, attributable to IL-24 because type 2 cytokine-induced IL-24 downregulates FLG and LOR expression via STAT3 [7, 9]. Depletion of IL-24 significantly restored the IL-13-induced downregulation of FLG [9]. Calmodulin-like 5 (CALML5) was revealed to be highly expressed in the differentiating epidermis. CALML5-knockout keratinocytes exhibit deficient FLG and LOR expression [58]. It was reported that IL-4 and 
IL-13 pathways converged on p63 to diminish CALML5 and FLG expression [59]. Interestingly, because IL-24 was also illustrated to inhibit the gene expression of CALML5 [39], the possible involvement of p63 in IL-24 signaling needs to be further studied. Collectively, these results indicate that IL-24 is a pivotal mediator of abnormal epidermal differentiation downstream of type 2 cytokine signals in AD.

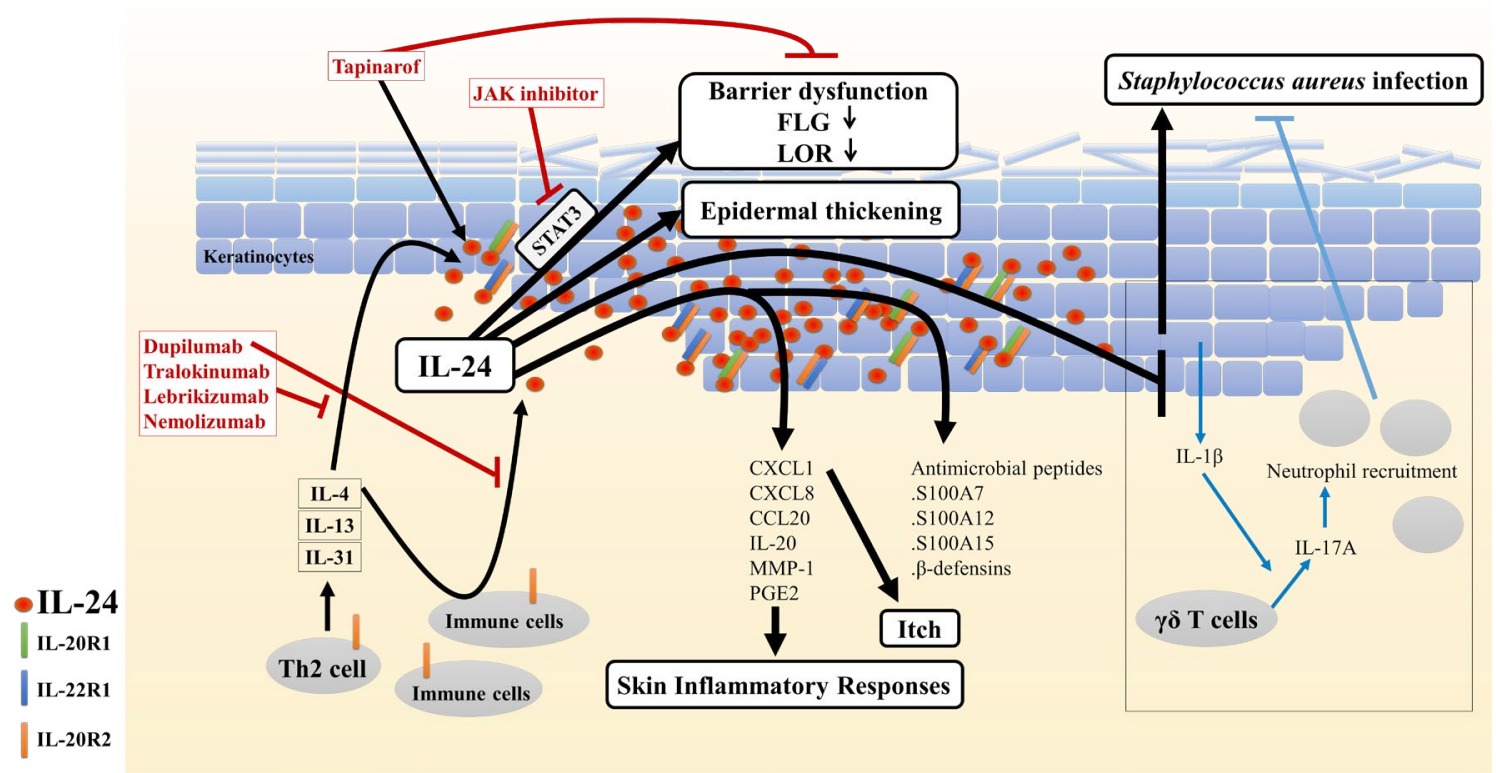

Figure 2. Potential role of IL-24 in AD. Type 2 cytokines stimulate immune cells and keratinocytes to secrete IL-24, leading to the upregulation of IL-24 expression in the epidermis of AD skin. Furthermore, IL-24 contributes to skin barrier disruption and hyperplasia in $\mathrm{AD}$, and it may exacerbate skin inflammatory responses, itch, and $S$. aureus infection. Therefore, therapeutics that suppress IL-24 signaling, including the IL-4/IL-13 antagonist dupilumab, IL-13 antagonists tralokinumab and lebrikizumab, IL-31Ra antagonist nemolizumab, and JAK-STAT inhibitors, might be promising for managing AD, including combination use with tapinarof

Common inflammatory skin diseases, including AD, feature keratinocyte hyperproliferation [60, 61]. IL24 was revealed to induce normal human epidermal keratinocyte proliferation in an epidermal growth factor receptor-independent manner, as blockade of this receptor in the monolayer culture system did not inhibit IL-24-mediated keratinocyte proliferation. However, keratinocyte proliferation might be further augmented through the upregulation of the EGF family ligands amphiregulin and heparin-binding epidermal growth factor induced by IL-24. IL-24 also increased the thickness of the reconstituted human epidermis [54]. Histological analysis revealed that IL-24 transgenic mice had a compact stratum corneum and markedly thickened epidermis compared with the findings in wild-type mice [42]. Additionally, IL24 was reported to contribute to epidermal hyperplasia induced by IL-23, a major cytokine implicated in psoriasis, in mice [41]. Furthermore, expression of the epidermal proliferation-associated proteins (keratin 16 and S100As, particularly S100A7) was increased in IL-24-treated reconstituted human epidermis [54].

In summary, these data suggest that IL-24 induces abnormal epidermal differentiation and hyperplasia, contributing to skin barrier dysfunction in AD.

\section{IL-24 and skin inflammatory responses}

IL-24 might participate in a complex cascade of cytokines involved in skin inflammatory responses, as IL-24 can induce the expression of several cytokines and chemokines. When added to cultured human keratinocytes and/or reconstituted human epidermis, IL-24 upregulated the expression of many inflammatory mediators, including chemokine (C-X-C motif) ligand (CXCL) 1 and IL-20 gene expression and CXCL8/IL-8, chemokine (C-C motif) ligand (CCL)20/macrophage inflammatory protein (MIP)-3 $\alpha$, matrix metalloproteinase (MMP)-1, and prostaglandin E2 (PGE2) protein secretion [15, 40, 52, 54]. Notably, $I L-24$ transgenic epidermis exhibited elevated induction of monocyte chemoattractant protein-1, a critical chemokine that mediates recruitment of monocytes/macrophages [42]. Furthermore, it was reported that $I L$-24-deficient mice were partially protected against allergic contact dermatitis and epidermal inflammatory infiltrate, particularly neutrophils, 
after exposure to paraphenylenediamine [16]. Altogether, these results indicate that IL-24 can promote skin inflammation, thereby contributing to the maintenance and exacerbation of AD.

\section{IL-24 and itch}

Intense itch induces scratching and skin lesion exacerbation that disturbs the quality of life of patients with AD [62]. The role of IL-24 in pruritus remains poorly explored. IL-31, a major pruritogenic cytokine in AD [62], induced $I L-24$ gene expression in keratinocytes [35]. IL-24 acts as an activator of STAT3, which is also activated by IL-31 to promote elongation of the nerve fibers, followed by enhanced itching [63]. Additionally, IL-24 upregulated the expression of CXCL1 [54], a chemokine that can evoke itch through multiple pathways $[64,65]$. These data suggest the involvement of IL-24 in the mechanism of pruritus in AD.

\section{IL-24 and skin infection}

The skin of patients with $\mathrm{AD}$ is prone to microbial infections [66]. Atopic skin is preferentially colonized by S. aureus, and its colonization further contributes to disease exacerbation [67, 68]. Myles et al. [25] reported that $S$. aureus-induced IL-24 inhibits the induction of IL-1 $\beta$ in keratinocytes, followed by a decrease in IL-17A expression in $\gamma \delta$ T cells, leading to reduced neutrophil recruitment and eventually resulting in more severe infection. They also suggested that IL-24 can promote cutaneous infection by $S$. aureus and that IL-20R2 blockade may have therapeutic potential for patients with $S$. aureus infection [25]. Conversely, in the human epidermis system, IL-24 was illustrated to induce several antimicrobial peptides that have a protective role against microbial infection, including S100A7, S100A12, S100A15, and $\beta$-defensins [52, 54]. These controversial findings imply that the anti-infection effects of IL-24 may vary depending on the infection conditions and timing.

\section{IL-24 and therapeutics for AD}

The aforementioned evidence suggests that IL-24 plays a significant role in the pathogenesis of AD and supports the potential efficacy of therapeutics that block the upstream and downstream pathways of IL24 signaling. Dupilumab, an anti-IL-4R $\alpha$ monoclonal antibody that inhibits IL-4 and IL-13 signaling, is efficacious in the treatment of moderate-to-severe AD. The drug significantly improves the clinical severity of AD. In parallel, the upregulation of $I L-24$ is suppressed in the lesional and non-lesional skin of patients with $\mathrm{AD}$ after 4 and 16 weeks of treatment with dupilumab [69]. It remains unclear whether other emerging drugs such as the IL-13 antibodies tralokinumab and lebrikizumab [70-72] and anti-IL-31R $\alpha$ antibody nemolizumab [70, 73] affect IL-24 expression in AD.

JAK inhibitors, such as topical delgocitinib and oral baricitinib, have demonstrated efficacy against AD, mainly owing to their inhibitory action on JAK/STAT signaling, and they also possess the potential to inhibit the IL-24 signaling [74-76].

Tapinarof has been illustrated to attenuate the disease activity of AD in clinical studies [4-6]. Our recent study demonstrated that tapinarof upregulates FLG and LOR expression in an AHR-dependent manner in human keratinocytes, thereby improving barrier dysfunction in AD. Paradoxically, tapinarof induced the secretion of IL-24, and IL-24 sequentially activates JAK-STAT3 signaling, which negatively regulates FLG and LOR expression, partially via AHR activation. Therefore, inhibition of the IL-24-STAT3 axis during AHR activation using JAK inhibitors may further increase FLG and LOR expression induced by tapinarof treatment. Therefore, co-treatment of tapinarof with JAK a inhibitor may be a promising strategy for managing AD [7].

\section{Conclusion}

IL-24 is an integral part of the pathogenic pivotal cytokine network in AD. Type 2 cytokines stimulate immune cells and keratinocytes to secrete IL-24. IL-24 contributes to epidermal barrier disruption and hyperplasia. By inducing several inflammatory mediators, IL-24 may orchestrate and stimulate skin inflammation and itch. Additionally, IL-24 may promote cutaneous infection by $S$. aureus. These findings underpin the fundamental role of IL-24 in the development of AD. Targeting the upstream and downstream pathways of IL-24 signaling represents a potential strategy for treating $\mathrm{AD}$. 


\section{Abbreviations}

AD: atopic dermatitis

AHR: aryl hydrocarbon receptor

CALML5: calmodulin-like 5

CXCL: chemokine (C-X-C motif) ligand

ERK: extracellular signal-regulated kinase

FLG: filaggrin

IL-24: interleukin-24

JAK: Janus kinase

LOR: loricrin

MAPK: mitogen-activated protein kinase

NF- $\kappa B$ : nuclear factor kappa-light-chain-enhancer of activated B cells

SOCS3: suppressor of cytokine signaling-3

STAT: signal transducer and activator of transcription

Th2: T helper 2

TNF: tumor necrosis factor

\section{Declarations}

\section{Author contributions}

YHV wrote the first draft of the manuscript. GT and MF reviewed the manuscript. All authors contributed to manuscript revision, and all authors read and approved the submitted version.

\section{Conflicts of interest}

The authors declare that they have no conflicts of interest.

\section{Ethical approval}

Not applicable.

\section{Consent to participate}

Not applicable.

\section{Consent to publication}

Not applicable.

Availability of data and materials

Not applicable.

\section{Funding}

Not applicable.

\section{Copyright}

(C) The Author(s) 2021.

\section{References}

1. Weidinger S, Novak N. Atopic dermatitis. Lancet. 2016;387:1109-22. 
2. Furue M, Ulzii D, Vu YH, Tsuji G, Kido-Nakahara M, Nakahara T. Pathogenesis of atopic dermatitis: current paradigm. Iran J Immunol. 2019;16:97-107.

3. Kim BE, Leung DYM. Significance of skin barrier dysfunction in atopic dermatitis. Allergy Asthma Immunol Res. 2018;10:207-15.

4. Bissonnette R, Chen G, Bolduc C, Maari C, Lyle M, Tang L, et al. Efficacy and safety of topical WBI-1001 in the treatment of atopic dermatitis: results from a phase $2 \mathrm{~A}$, randomized, placebo-controlled clinical trial. Arch Dermatol. 2010;146:446-9.

5. Bissonnette R, Poulin Y, Zhou Y, Tan J, Hong HC, Webster J, et al. Efficacy and safety of topical WBI-1001 in patients with mild to severe atopic dermatitis: results from a 12-week, multicentre, randomized, placebocontrolled double-blind trial. Br J Dermatol. 2012;166:853-60.

6. Peppers J, Paller AS, Maeda-Chubachi T, Wu S, Robbins K, Gallagher K, et al. A phase 2, randomized dose-finding study of tapinarof (GSK2894512 cream) for the treatment of atopic dermatitis. J Am Acad Dermatol. 2019;80:89-98.e3.

7. Vu YH, Hashimoto-Hachiya A, Takemura M, Yumine A, Mitamura Y, Nakahara T, et al. IL-24 negatively regulates keratinocyte differentiation induced by tapinarof, an aryl hydrocarbon receptor modulator: implication in the treatment of atopic dermatitis. Int J Mol Sci. 2020;21:9412.

8. Bao L, Zhang H, Mohan GC, Shen K, Chan LS. Differential expression of inflammation-related genes in IL-4 transgenic mice before and after the onset of atopic dermatitis skin lesions. Mol Cell Probes. 2016;30:30-8.

9. Mitamura Y, Nunomura S, Nanri Y, Ogawa M, Yoshihara T, Masuoka M, et al. The IL-13/periostin/IL-24 pathway causes epidermal barrier dysfunction in allergic skin inflammation. Allergy. 2018;73:1881-91.

10. Menezes ME, Bhoopathi P, Pradhan AK, Emdad L, Das SK, Guo C, et al. Role of MDA-7/IL-24 a multifunction protein in human diseases. Adv Cancer Res. 2018;138:143-82.

11. Mitamura Y, Nunomura S, Furue M, Izuhara K. IL-24: a new player in the pathogenesis of pro-inflammatory and allergic skin diseases. Allergol Int. 2020;69:405-11.

12. Tsoi LC, Rodriguez E, Degenhardt F, Baurecht H, Wehkamp U, Volks N, et al. Atopic dermatitis is an IL-13-dominant disease with greater molecular heterogeneity compared to psoriasis. J Invest Dermatol. 2019;139:1480-9.

13. Kitamura A, Takata R, Aizawa S, Watanabe H, Wada T. A murine model of atopic dermatitis can be generated by painting the dorsal skin with hapten twice 14 days apart. Sci Rep. 2018;8:5988.

14. Kunz S, Wolk K, Witte E, Witte K, Doecke WD, Volk HD, et al. Interleukin (IL)-19, IL-20 and IL-24 are produced by and act on keratinocytes and are distinct from classical ILs. Exp Dermatol. 2006;15:991-1004.

15. Jin SH, Choi D, Chun YJ, Noh M. Keratinocyte-derived IL-24 plays a role in the positive feedback regulation of epidermal inflammation in response to environmental and endogenous toxic stressors. Toxicol Appl Pharmacol. 2014;280:199-206.

16. Van Belle AB, Cochez PM, de Heusch M, Pointner L, Opsomer R, Raynaud P, et al. IL-24 contributes to skin inflammation in Para-Phenylenediamine-induced contact hypersensitivity. Sci Rep. 2019;9:1852.

17. Tang KW, Lin ZC, Chen YL, Tzeng CC, Fang JY, Tseng CH. Synthesis and biological evaluation of thalidomide derivatives as potential anti-psoriasis agents. Int J Mol Sci. 2018;19:3061.

18. Liu G, Asanoma K, Takao T, Tsukimori K, Uchi H, Furue M, et al. Aryl hydrocarbon receptor SNP-130 C/T associates with dioxins susceptibility through regulating its receptor activity and downstream effectors including interleukin 24. Toxicol Lett. 2015;232:384-92.

19. Luo YH, Kuo YC, Tsai MH, Ho CC, Tsai HT, Hsu CY, et al. Interleukin-24 as a target cytokine of environmental aryl hydrocarbon receptor agonist exposure in the lung. Toxicol Appl Pharmacol. 2017;324:1-11. 
20. Sun H, Shamy M, Kluz T, Muñoz AB, Zhong M, Laulicht F, et al. Gene expression profiling and pathway analysis of human bronchial epithelial cells exposed to airborne particulate matter collected from Saudi Arabia. Toxicol Appl Pharmacol. 2012;265:147-57.

21. Heinemann A, He Y, Zimina E, Boerries M, Busch H, Chmel N, et al. Induction of phenotype modifying cytokines by FERMT1 mutations. Hum Mutat. 2011;32:397-406.

22. Smith SH, Jayawickreme C, Rickard DJ, Nicodeme E, Bui T, Simmons C, et al. Tapinarof is a natural AhR agonist that resolves skin inflammation in mice and humans. J Invest Dermatol. 2017;137:2110-9.

23. Sun YV, Boverhof DR, Burgoon LD, Fielden MR, Zacharewski TR. Comparative analysis of dioxin response elements in human, mouse and rat genomic sequences. Nucleic Acids Res. 2004;32:4512-23.

24. Buzas K, Megyeri K. Staphylococci induce the production of melanoma differentiation-associated protein-7/IL-24. Acta Microbiol Immunol Hung. 2006;53:431-40.

25. Myles IA, Fontecilla NM, Valdez PA, Vithayathil PJ, Naik S, Belkaid Y, et al. Signaling via the IL-20 receptor inhibits cutaneous production of IL-1 $\beta$ and IL-17A to promote infection with methicillin-resistant Staphylococcus aureus. Nat Immunol. 2013;14:804-11.

26. Rutz S, Wang X, Ouyang W. The IL-20 subfamily of cytokines--from host defence to tissue homeostasis. Nat Rev Immunol. 2014;14:783-95.

27. Garn H, Schmidt A, Grau V, Stumpf S, Kaufmann A, Becker M, et al. IL-24 is expressed by rat and human macrophages. Immunobiology. 2002;205:321-34.

28. Wang M, Liang P. Interleukin-24 and its receptors. Immunology. 2005;114:166-70.

29. Kim S, Lee SY, Bae S, Lee JK, Hwang K, Go H, et al. Pellino1 promotes chronic inflammatory skin disease via keratinocyte hyperproliferation and induction of the T helper 17 response. Exp Mol Med. 2020;52:1537-49.

30. Sugaya M. The role of Th17-related cytokines in atopic dermatitis. Int J Mol Sci. 2020;21:1314.

31. Wei L, Vahedi G, Sun HW, Watford WT, Takatori H, Ramos HL, et al. Discrete roles of STAT4 and STAT6 transcription factors in tuning epigenetic modifications and transcription during T helper cell differentiation. Immunity. 2010;32:840-51.

32. Dabitao D, Hedrich CM, Wang F, Vacharathit V, Bream JH. Cell-specific requirements for STAT proteins and Type I IFN receptor signaling discretely regulate IL-24 and IL-10 expression in NK cells and macrophages. J Immunol. 2018;200:2154-64.

33. Sahoo A, Lee CG, Jash A, Son JS, Kim G, Kwon HK, et al. Stat6 and c-Jun mediate Th2 Cell-Specific IL-24 gene expression. J Immunol. 2011;186:4098-109.

34. Stevens L, Htut TM, White D, Li X, Hanidu A, Stearns C, et al. Involvement of GATA3 in protein kinase C $\theta$-induced Th2 cytokine expression. Eur J Immunol. 2006;36:3305-14.

35. Cornelissen C, Marquardt Y, Czaja K, Wenzel J, Frank J, Lüscher-Firzlaff J, et al. IL-31 regulates differentiation and filaggrin expression in human organotypic skin models. J Allergy Clin Immunol. 2012;129:426-33, 433.e1-8.

36. Wang F, Smith N, Maier L, Xia W, Hammerberg C, Chubb H, et al. Etanercept suppresses regenerative hyperplasia in psoriasis by acutely downregulating epidermal expression of interleukin (IL)-19, IL-20 and IL-24. Br J Dermatol. 2012;167:92-102.

37. Chong WP, Mattapallil MJ, Raychaudhuri K, Bing SJ, Wu S, Zhong Y, et al. The cytokine IL-17A limits Th17 pathogenicity via a negative feedback loop driven by autocrine induction of IL-24. Immunity. 2020;53:384-397.e5.

38. Konrad RJ, Higgs RE, Rodgers GH, Ming W, Qian YW, Bivi N, et al. Assessment and clinical relevance of serum IL-19 levels in psoriasis and atopic dermatitis using a sensitive and specific novel immunoassay. Sci Rep. 2019;9:5211. 
39. Martin JC, Wolk K, Bériou G, Abidi A, Witte-Händel E, Louvet C, et al. Limited presence of IL-22 binding protein, a natural IL-22 inhibitor, strengthens psoriatic skin inflammation. J Immunol. 2017;198:3671-8.

40. Tohyama M, Hanakawa Y, Shirakata Y, Dai X, Yang L, Hirakawa S, et al. IL-17 and IL-22 mediate IL-20 subfamily cytokine production in cultured keratinocytes via increased IL-22 receptor expression. Eur J Immunol. 2009;39:2779-88.

41. Chan JR, Blumenschein W, Murphy E, Diveu C, Wiekowski M, Abbondanzo S, et al. IL-23 stimulates epidermal hyperplasia via TNF and IL-20R2-dependent mechanisms with implications for psoriasis pathogenesis. J Exp Med. 2006;203:2577-87.

42. He M, Liang P. IL-24 transgenic mice: in vivo evidence of overlapping functions for IL-20, IL-22, and IL-24 in the epidermis. J Immunol. 2010;184:1793-8.

43. Andoh A, Shioya M, Nishida A, Bamba S, Tsujikawa T, Kim-Mitsuyama S, et al. Expression of IL-24, an activator of the JAK1/STAT3/SOCS3 cascade, is enhanced in inflammatory bowel disease. J Immunol. 2009;183:687-95.

44. Otkjaer K, Holtmann H, Kragstrup TW, Paludan SR, Johansen C, Gaestel M, et al. The p38 MAPK regulates IL-24 expression by stabilization of the 3' UTR of IL-24 mRNA. PLoS One. 2010;5:e8671.

45. Azuma YT, Matsuo Y, Nakajima H, Yancopoulos GD, Valenzuela DM, Murphy AJ, et al. Interleukin-19 is a negative regulator of innate immunity and critical for colonic protection. J Pharmacol Sci. 2011;115:105-11.

46. Ko YK, An SJ, Han NY, Lee H, Choi BK. Regulation of IL-24 in human oral keratinocytes stimulated with Tannerella forsythia. Mol Oral Microbiol. 2019;34:209-18.

47. Carow B, Rottenberg ME. SOCS3, a major regulator of infection and inflammation. Front Immunol. 2014;5:58.

48. Uto-Konomi A, Miyauchi K, Ozaki N, Motomura Y, Suzuki Y, Yoshimura A, et al. Dysregulation of suppressor of cytokine signaling 3 in keratinocytes causes skin inflammation mediated by interleukin-20 receptor-related cytokines. PLoS One. 2012;7:e40343.

49. Tang KW, Lin ZC, Wang PW, Alalaiwe A, Tseng CH, Fang JY. Facile skin targeting of a thalidomide analog containing benzyl chloride moiety alleviates experimental psoriasis via the suppression of MAPK/NFкB/AP-1 phosphorylation in keratinocytes. J Dermatol Sci. 2020;99:90-9.

50. Weng JR, Huang TH, Lin ZC, Alalaiwe A, Fang JY. Cutaneous delivery of [1-(4-chloro-3nitrobenzenesulfonyl)-1H-indol-3-yl]-methanol, an indole-3-carbinol derivative, mitigates psoriasiform lesion by blocking MAPK/NF-кB/AP-1 activation. Biomed Pharmacother. 2019;119:109398.

51. Fujita T, Matsuoka T, Honda T, Kabashima K, Hirata T, Narumiya S. A GPR40 agonist GW9508 suppresses CCL5, CCL17, and CXCL10 induction in keratinocytes and attenuates cutaneous immune inflammation. J Invest Dermatol. 2011;131:1660-7.

52. Kumari S, Bonnet MC, Ulvmar MH, Wolk K, Karagianni N, Witte E, et al. Tumor necrosis factor receptor signaling in keratinocytes triggers interleukin-24-dependent psoriasis-like skin inflammation in mice. Immunity. 2013;39:899-911.

53. Niess JH, Hruz P, Kaymak T. The interleukin-20 cytokines in intestinal diseases. Front Immunol. 2018;9:1373.

54. Sa SM, Valdez PA, Wu J, Jung K, Zhong F, Hall L, et al. The effects of IL-20 subfamily cytokines on reconstituted human epidermis suggest potential roles in cutaneous innate defense and pathogenic adaptive immunity in psoriasis. J Immunol. 2007;178:2229-40.

55. Rømer J, Hasselager E, Nørby PL, Steiniche T, Clausen JT, Kragballe K. Epidermal overexpression of interleukin-19 and -20 mRNA in psoriatic skin disappears after short-term treatment with cyclosporine A or calcipotriol. J Invest Dermatol. 2003;121:1306-11. 
56. Sabat R, Wallace E, Endesfelder S, Wolk K. IL-19 and IL-20: two novel cytokines with importance in inflammatory diseases. Expert Opin Ther Targets. 2007;11:601-12.

57. Egawa G, Kabashima K. Barrier dysfunction in the skin allergy. Allergol Int. 2018;67:3-11.

58. Sun BK, Boxer LD, Ransohoff JD, Siprashvili Z, Qu K, Lopez-Pajares V, et al. CALMI5 is a ZNF750- and TINCRinduced protein that binds stratifin to regulate epidermal differentiation. Genes Dev. 2015;29:2225-30.

59. Brauweiler AM, Leung DYM, Goleva E. The transcription factor p63 is a direct effector of IL-4- and IL13-mediated repression of keratinocyte differentiation. J Invest Dermatol. 2020;[Epub ahead of print].

60. Cibrian D, de la Fuente H, Sánchez-Madrid F. Metabolic pathways that control skin homeostasis and inflammation. Trends Mol Med. 2020;26:975-86.

61. Esaki H, Brunner PM, Renert-Yuval Y, Czarnowicki T, Huynh T, Tran G, et al. Early-onset pediatric atopic dermatitis is TH2 but also TH17 polarized in skin. J Allergy Clin Immunol. 2016;138:1639-51.

62. Furue K, Ulzii D, Tanaka Y, Ito T, Tsuji G, Kido-Nakahara M, et al. Pathogenic implication of epidermal scratch injury in psoriasis and atopic dermatitis. J Dermatol. 2020;47:979-88.

63. Feld M, Garcia R, Buddenkotte J, Katayama S, Lewis K, Muirhead G, et al. The pruritus- and TH2-associated cytokine IL-31 promotes growth of sensory nerves. J Allergy Clin Immunol. 2016;138:500-8.e24.

64. Walsh CM, Hill RZ, Schwendinger-Schreck J, Deguine J, Brock EC, Kucirek N, et al. Neutrophils promote CXCR3-dependent itch in the development of atopic dermatitis. Elife. 2019;8:e48448.

65. Deftu AF, Filippi A, Gheorghe RO, Ristoiu V. CXCL1 activates TRPV1 via Gi/o protein and actin filaments. Life Sci. 2018;193:282-91.

66. Guttman-Yassky E, Nograles KE, Krueger JG. Contrasting pathogenesis of atopic dermatitis and psoriasis--part II: immune cell subsets and therapeutic concepts. J Allergy Clin Immunol. 2011;127:1420-32.

67. Furue M, Iida K, Imaji M, Nakahara T. Microbiome analysis of forehead skin in patients with atopic dermatitis and healthy subjects: implication of Staphylococcus and Corynebacterium. J Dermatol. 2018;45:876-7.

68. Rangel SM, Paller AS. Bacterial colonization, overgrowth, and superinfection in atopic dermatitis. Clin Dermatol. 2018;36:641-7.

69. Guttman-Yassky E, Bissonnette R, Ungar B, Suárez-Fariñas M, Ardeleanu M, Esaki H, et al. Dupilumab progressively improves systemic and cutaneous abnormalities in patients with atopic dermatitis. J Allergy Clin Immunol. 2019;143:155-72.

70. Nguyen HL, Anderson KR, Tollefson MM. New and emerging therapies for pediatric atopic dermatitis. Pediatr Drugs. 2019;21:239-60.

71. Wollenberg A, Howell MD, Guttman-Yassky E, Silverberg JI, Kell C, Ranade K, et al. Treatment of atopic dermatitis with tralokinumab, an anti-IL-13 mAb. J Allergy Clin Immunol. 2019;143:135-41.

72. Simpson EL, Flohr C, Eichenfield LF, Bieber T, Sofen H, Taïeb A, et al. Efficacy and safety of lebrikizumab (an anti-IL-13 monoclonal antibody) in adults with moderate-to-severe atopic dermatitis inadequately controlled by topical corticosteroids: a randomized, placebo-controlled phase II trial (TREBLE). J Am Acad Dermatol. 2018;78:863-71.e11.

73. Nemoto O, Furue M, Nakagawa H, Shiramoto M, Hanada R, Matsuki S, et al. The first trial of CIM331, a humanized antihuman interleukin-31 receptor A antibody, in healthy volunteers and patients with atopic dermatitis to evaluate safety, tolerability and pharmacokinetics of a single dose in a randomized, double-blind, placebo-controlled study. Br J Dermatol. 2016;174:296-304.

74. Solimani F, Meier K, Ghoreschi K. Emerging topical and systemic JAK inhibitors in dermatology. Front Immunol. 2019;10:2847. 
75. Simpson EL, Lacour JP, Spelman L, Galimberti R, Eichenfield LF, Bissonnette R, et al. Baricitinib in patients with moderate-to-severe atopic dermatitis and inadequate response to topical corticosteroids: results from two randomized monotherapy phase III trials. Br J Dermatol. 2020;183:242-55.

76. Nakagawa H, Nemoto O, Igarashi A, Saeki H, Kaino H, Nagata T. Delgocitinib ointment, a topical Janus kinase inhibitor, in adult patients with moderate to severe atopic dermatitis: a phase 3, randomized, double-blind, vehicle-controlled study and an open-label, long-term extension study. J Am Acad Dermatol. 2020;82:823-31. 